\title{
Emerging Contaminants: Extracting Micro \& Nanomaterial Data from Soil Samples
}

Katrina Varner

US Environmental Protection Agency, Office of Research and Development, National Exposure Research Laboratory, Exposure Methods and Measurement Division 109 TW Alexander Drive, Research Triangle Park, NC 27711 USA

The EPA is expounding upon various exposure methods involving new and emerging contaminants of concern to increasing discoveries of risk analysis and management of environmental events. These concerns involve micro and nanomaterials, which may start as engineered material that interact with known and unknown substances in the environment. The advancement of these novel materials and emerging compounds in the commercial sector has facilitated vast improvements with modern technology. Many of these innovative chemical compounds have resulted in enhanced quality of life which includes sophisticated medicinal applications, decreasing energy needs and increase of computational power. However, their environmental impacts especially as considered through the entire material assessment, are still both poorly researched and inadequately assessed. There are many challenges in protecting the environment as materials are developed and used, including the prevention of unintended consequences of exposures to humans and the ecosystem. [1] Understanding the long-term health and environmental effects of these newly formed materials and communicating with the public about exposure, risks and benefits [2] is work accomplished at the National Research Exposure Laboratory and presented here.

To evaluate the effects of nanomaterials on the environment and human health, considerable knowledge of the nature and properties of the nanomaterials are required. [3] Relevant chemical and physical properties that could be measured include characteristics as purity, particle size and distribution, shape, crystal structure, composition, surface area, surface chemistry, surface charge, surface activity, and porosity. The environmental transport of materials depends upon environmental media, parameters and interactions. This study addresses the concern of (a) identifying the transport of (b) buckminsterfullerene micro and nanomaterials with the binding affinity to metals weathered [4] in soil via scanning electron microscopy $[5,6]$.

A $1 \mathrm{mM}(0.7205 \mathrm{~g})$ stock solution of buckminsterfullerene $\left(\mathrm{C}_{60}\right)$ in toluene was prepared in a $1 \mathrm{~L}$ volumetric flask and used as base solution for all ensuing samples. The flask was placed in a water bath shaker set at $29.0^{\circ} \mathrm{C}$ and operating at 180 RPM for 72 hours. At the end of the incubation time, the mixture turns into a deep purple color indicating that the $\mathrm{C}_{60}$ material has dissolved into the toluene. Soil samples (sand, clay, silt, loam and peat) from around the United States were spiked with the $\mathrm{C}_{60}$ solution and placed in a Q-Sun ${ }^{\circledR}$ Weathering System. The weathering system was programed for simulation of a month of various climate considerations including extreme heat as in the southwest and moisture to simulate rain. The conditioned soil samples were then loaded on a Dionex ${ }^{\circledR}$ Accelerated Solvent Extraction (ASE) System where they were eluted using a combination of methanol and toluene to collect fullerene materials. Soil extracts $(40 \mathrm{~mL})$ were concentrated down to $2 \mathrm{~mL}$ using a Dionex ${ }^{\circledR}$ Solvent Evaporator (SE). The sample were then mounted on PELCO ${ }^{\circledR}$ Support Films for STEM/TEM. These were analyzed with the Zeiss SIGMA-VP Field Emission Scanning Electron Microscope STEM Stage with: 
Lacey Formvar/Carbon, 200 mesh, Copper; Lacey Formvar/Carbon, 300 mesh, Copper; and Lacey Carbon Type A, 300 mesh, Copper. The SEM specimen grids were examined via SE2, InLens, and STEM with energy dispersive X-ray analysis (EDX) to determine if metal was present.

Results of the weathered $\mathrm{C}_{60}$ experiment show that the fullerenes do interact or to be more specific bind with metals found in the soil samples and this, regardless of soil types. This suggest that plants grown on soils contaminated with fullerenes could have carbon and metal-based micro and nanoparticles available for uptake, which should be of environmental concern. It is the mission of the Environmental Protection Agency to address the many challenges in protecting the environment from harmful results of micro and nanomaterials as these materials are being used and developed from the unintended consequences including exposure and ill-fated risks to humans and the ecosystem. With the explosive growth in the applications of engineered micro and nanomaterials, there is also a likewise explosive growth of implications for new and emerging products, which lead to the many challenges of protecting the environment. The applied solution to the challenge of recognizing binding affinity and uptake of micro and nanomaterial has been identified via STEM SEM imaging. [7]

NOTICE: The United States Environmental Protection Agency through its Office of Research and Development performed this research. It has been subjected to Agency review and approved for publication. Although this work was reviewed by U.S. EPA and approved for publication, it may not necessarily reflect official Agency policy. Mention of trade names or commercial products does not constitute endorsement or recommendation for use.

References:

[1] Wang, Junfeng; et.al. "Observation of Fullerene Soot in Eastern China," Environmental Science and Technology, Volume 3, Pages 121-126, March 4, 2016.

[2] Turley, Andrew. "OECD Conclusions About Nanomaterials and Test Guidelines Disputed," Chemical Watch, Global Risk and Regulation News, 5 January 2017

[3] Batista, C.A.S.; Larson, R.G.; and Kotov, N.A. "Nonadditivity of Nanoparticle Interactions," Science, Volume 350, Page 176, 2015.

[4] Wohlleben, Wendel; et al. "NanoRelease: Pilot Interlaboratory Comparison of a Weathering Protocol Applied to Resilient and Labile Polymers with and without Embedded Carbon Nanotubes," Carbon, Volume 113, Pages 346-360, March 2017

[5] Goldstein, Joseph I.; et al. Scanning Electron Microscopy and X-ray Microanalysis, Springer, 2003

[6] Kiessling, F.; Mertens, M.E.; Grimm, J; and Lammers, T. "Nanoparticles for Imaging: Top or Flop?” Radiology, Volume 273 (1), Pages 10-28.

[7] Acknowledgements: The author thanks the many EPA students, Jeremy Hilgar, Darlene Usi, Shane Maheffey, and Carrie Garrison, who have worked on this project. 